\title{
A pesquisa na sala de aula de matemática das séries iniciais do ensino fundamental. Contribuições teóricas da psicologia
}

\section{Research inside the primary school mathematics classroom. Theoretical Contributions from Psychology}

\author{
Sandra Magina ${ }^{1}$
}

\begin{abstract}
RESUMO
O presente artigo inicia pela discussão sobre as contribuições que pesquisadores de diversas áreas - psicologia, história, matemática, antropologia, entre outras - trazem para a Educação Matemática. A ênfase será nas pesquisas que tomam por base a psicologia, detendo-se na teoria dos campos conceituais. Para ilustrar sua posição, o artigo apresenta um estudo descritivo realizado com 103 professoras polivalentes que atuam nas séries iniciais do Ensino Fundamental, no qual foi pedido para que elas elaborassem quatro problemas de estrutura aditiva. Os resultados são analisados à luz da referida teoria e apontam que a maioria dos problemas elaborados $(88,43 \%)$ são problemas considerados como protótipos; ou seja, os menos complexos cognitivamente. $\mathrm{O}$ estudo conclui que parece não haver uma preocupação, ou compreensão, dos professores em trabalhar problemas que facilitem o entendimento e a expansão do campo conceitual aditivo de seus estudantes.

Palavras-chave: estruturas aditivas; professores polivalentes; estudo descritivo; psicologia cognitiva; educação matemática.
\end{abstract}

1 Sandra Magina é $\mathrm{PhD}$ pela Universidade de Londres e pós-doutora pela Universidade de Lisboa, professora do Programa de Pós-Graduação em Educação Matemática da Pontifícia Universidade Católica de São Paulo - PUC/SP Brasil - sandra@pucsp.br 


\begin{abstract}
The starting point of this paper is to promote a discussion concerning the contributions that were brought to Mathematics Education from researchers from different areas, such as Psychology, Mathematics, Anthropology, History, among others. The focus will be on research based on Psychology, in special on the theory of conceptual field (TCF). To illustrate this position, the paper presents a descriptive study carried out with 103 primary school teachers, in which they were asked to elaborate four problems of addictive structure. The results, which are analyzed from the TCF perspective, point out that most of these elaborated problems $(88,43 \%)$ were classified as prototypes ones, that is, those which require less cognitive effort. The study concludes that it seems that there is no worrying, or comprehension, from teachers in relation to explore problems that make their students' understanding easier or to expand the addictive conceptual field.

Keywords: addictive structures; primary school teachers; descriptive study; cognitive psychology; mathematics education.
\end{abstract}

\title{
Introdução
}

Uma das grandes características da educação matemática é a sua pluralidade, o que exige a presença de pesquisadores com formações iniciais distintas, tais como matemática, filosofia, antropologia, sociologia, história, pedagogia e psicologia, para citar apenas algumas das possibilidades. A consequência positiva desse sincretismo científico é a produção de distintos tipos de pesquisas, abordando as múltiplas facetas da Educação Matemática. É justamente sobre os benefícios advindos desse sincretismo que versa o presente artigo.

\section{A benéfica pluralidade da Educação Matemática}

No que concerne às contribuições das pesquisas voltadas para os aspectos psicológicos, iniciamos apontando as produções de Piaget (PIAGET, 1995; PIAGET; INHELDER, 1961; 1995; PIAGET; SZEMINSKA, 1975), seguido pela produção de quem foi formado por ele no doutoramento, Vergnaud (1982; 1990; 1996; 1998; 2009); e, ainda, de quem se apoia nas teorias de ambos, tais como Carraher, Carraher e 
Schliemann (1989), Nunes e Bryant (1997), Nunes, Campos, Magina e Bryant (2005), Magina, Campos, Nunes e Gitirana (2001), Magina e Campos (2004), entre vários outros pesquisadores brasileiros. Nessa sequência cronológica, apontaria também Spinillo (2006). Spinillo e Magina (2004) e Meira (1996; 2002; 2003), alunos de mestrado de Nunes, além de Healy (HEALY; POZZI; SUTHERLAND, 2001; HEALY; SINCLAIR, 2007; FERNANDES; HEALY, 2008), cujos estudos em ambiente computacional têm expressão mundial. Todos esses são bons exemplos da pesquisa que toma por base teórica constructos psicológicos. De um modo geral, a pesquisa psicológica é desenvolvida fora da sala de aula, envolvendo um número pequeno de sujeitos. O foco principal é buscar explicações para o desenvolvimento do raciocínio matemático, ou, ainda, voltar-se para entender os fatores psicológicos que influenciam e/ou interferem na apropriação de um determinado conceito matemático.

Já as pesquisas produzidas por historiadores da educação matemática costumam se basear principalmente em documentos oficiais, em arquivos da mídia e em entrevistas. Os trabalhos liderados por Valente e Silva (2004), Valente (2009) e por Garnica e Baraldi (2005) e Garnica e Silva (2010) constituem amostra, com abordagens distintas, desse tipo de pesquisa. As pesquisas de cunho antropológico costumam ser realizadas em contextos cotidianos, fora do ambiente escolar, mas onde se apresentam situações matemáticas com as quais o indivíduo lida, mesmo sem ter consciência disso. Trabalhos como o de Lave (LAVE; WENGER, 1998) e de Carraher (CARRAHER; CARRAHER; SCHILIEMAN, 1989) são exemplos clássicos das pesquisas com foco antropológico.

Ainda vale ressaltar algumas das pesquisas feitas por educadores matemáticos, cuja formação primordial (graduação e pós-graduação) é na área de Matemática, as quais costumam seguir o viés teórico, priorizando o saber matemático. Assim sendo, mesmo quando propõem modelos de ensino, não se baseiam necessariamente em dados empíricos. Podemos encontrar um bom exemplo desse tipo de pesquisa em Machado (1992; 2003).

É importante, contudo, esclarecer que essas linhas de pesquisa não são tão demarcadas ou isoladas como pode parecer. Afinal, não podemos esquecer que estamos tratando de uma ciência que já nasceu interdisciplinar. Assim, temos Ubiratan D’Ambrósio (1993; 2001), grande matemático, que nas últimas décadas desenvolveu, e vem pesquisando, criando inclusive escola, a Etnomatemática. Esta tem vínculo com os conteúdos matemáticos, mas, igualmente, ao cotidiano extraescola das pessoas, portanto, muito ligado aos estudos antropológicos. Nessa mesma direção de interdisciplinaridade, temos Carraher et. al. (1989), cujos trabalhos com crianças que vendiam frutas nas ruas do Recife podiam ser enquadrados dentro da antropologia. Carraher, que a partir de 1995 assumiu o sobrenome de Nunes, também tem produzido pesquisas, tanto com crianças brasileiras, como inglesas, de caráter eminentemente psicológico (NUNES; BRYANT, 1997; NUNES et al., 2005). Faz-se necessário 
abrir um parêntese na linha do pensamento para ressaltar a importância desses dois últimos pesquisadores - D'Ambrósio e Nunes - no surgimento e desenvolvimento da Educação Matemática em nosso País.

Com esse espírito de interdisciplinaridade, há muitos grupos atuando fortemente no Brasil. Um desses grupos está na Pontifícia Universidade Católica de São Paulo (PUC/SP), onde atuo. De fato, a maioria das pesquisas que venho desenvolvendo no Programa de Pós-Graduação em Educação Matemática da PUC/SP é fruto do trabalho conjunto com colegas cuja formação é em matemática. Esses estudos são voltados para a sala de aula, mas nem todos são ou foram desenvolvidos $\underline{\mathrm{em}}$ sala de aula. Embora em algumas pesquisas tenha realizado um planejamento experimental, de intervenção, a maioria delas teve por objetivo descrever, registrar, analisar e correlacionar fatos ou fenômenos (do mundo humano), sem, contudo, manipulá-los. Isto é, foram pesquisas classificadas como descritivas (GIL, 2002; RUDIO, 2009), nas quais, como pesquisadora, não interferi (pelo menos não propositalmente) e/ou manipulei variáveis.

\section{A pesquisa descritiva}

As direções de pesquisas descritas acima são relevantes e essenciais para o desenvolvimento da Educação Matemática. Porém, para o propósito deste artigo, optei por abordar o tema discutindo apenas uma pesquisa realizada $\underline{\underline{e m}}$ sala de aula do Ensino Fundamental, cujo objetivo foi o de buscar estabelecer relações entre o saber dos alunos (medido por instrumentos oficiais da educação no Brasil) e dos professores que atuam nas séries iniciais do Ensino Fundamental.

As pesquisas $\underline{\boldsymbol{e m}}$ sala de aula apresentam, pelo menos, duas possibilidades de realização: as diagnósticas e as intervencionistas. As primeiras tendem a atuar por meio da aplicação de instrumentos de mensuração, e as segundas por meio de sequências (ou intervenção) de ensino.

Considerando as pesquisas diagnósticas, é importante distinguir aquelas que têm o caráter de avaliação do sistema das pesquisas realizadas pelos pesquisadores doutores, a maioria atuando em instituições de ensino superior, que respondem a questões pontuais. Em geral, as primeiras são realizadas por órgãos governamentais, como é o caso do SAEB - Sistema de Avaliação da Educação Básica - com amplitude nacional. Ambos os tipos de pesquisa são importantes e trazem imensas contribuições para a educação matemática. Porém são de essências diferentes: enquanto o primeiro tem por foco avaliar o sistema educacional brasileiro em determinados anos de escolarização ( $4 .^{\mathrm{a}}$ e 8 . $^{\mathrm{a}}$ séries) e não necessita de fundamentação teórica para apresentar seus resultados, o segundo busca, com base nos paradigmas 
teóricos e científicos, identificar os fenômenos que estão por trás da apropriação dos conceitos matemáticos e, por isso, algumas vezes estudam os alunos, outras, o professor. Este tipo de pesquisa serve de subsídio para as posteriores pesquisas experimentais, ou intervencionistas, já que pode oferecer ao pesquisador um bom panorama (ou fotografia) sobre uma determinada situação, fenômeno ou fato, o que lhe permitirá realizar uma pesquisa de intervenção mais acurada.

É esse o viés da pesquisa em educação matemática que será privilegiado no presente artigo. Apresentarei pesquisa, de cunho metodológico descritivo, cujos resultados serão discutidos à luz da teoria psicológica dos campos conceituais (VERGNAUD, 1996), a qual será brevemente apresentada a seguir.

\section{A teoria dos campos conceituais}

A teoria dos campos conceituais, formulada por Vergnaud (1982; 1990; 1996), é uma teoria psicológica consistente para pesquisas sobre atividades cognitivas, em especial, para aquelas de formação de conceitos matemáticos. Ela permite situar e estudar as filiações e as rupturas entre conhecimentos, o que implica estudar as teias de relação existentes entre os conceitos matemáticos. Em outras palavras, trata-se de uma teoria cognitiva que oferece um quadro coerente e alguns princípios de base para o estudo do desenvolvimento e da aprendizagem de competências complexas.

Essa teoria possibilita duas análises importantes: a primeira refere-se à relação entre os conceitos como conhecimentos explícitos e os invariantes operatórios implícitos nos comportamentos dos sujeitos frente à determinada situação, e a segunda, sustenta um aprofundamento das relações existentes entre significado e significante.

Assim, a teoria dos campos conceituais postula que os conceitos matemáticos tracem seus sentidos com base em uma variedade de situações e, normalmente, cada situação, não pode ser analisada com a ajuda de apenas um conceito. Em outras palavras, uma situação, por mais simples que seja, envolve mais que um conceito e, por outro lado, um conceito não pode ser apropriado a partir da vivência de uma única situação.

Por isso podemos nos referir a um campo conceitual como sendo um conjunto de problemas ou situações, cuja análise e tratamento requerem vários tipos de conceitos, procedimentos e representações simbólicas, os quais se encontram em estreita conexão uns com os outros.

A teoria dos campos conceituais fornece elementos para a análise das dificuldades dos alunos e se constitui em uma poderosa ferramenta para a construção de situações-problema. Isto porque ela apresenta um quadro coerente para o estudo do desenvolvimento e da aprendizagem de competências complexas. 
Em matemática, Vergnaud $(1982 ; 1990 ; 2009)$ aponta que dois campos conceituais são necessários para subsidiar todos os demais conceitos matemáticos: o campo conceitual das estruturas aditivas e o campo conceitual das estruturas multiplicativas. O primeiro se caracteriza como um conjunto de situações que requerem para a sua resolução uma operação de adição ou subtração ou as duas combinadas. O segundo se caracteriza como um conjunto de situações que requerem para a sua resolução uma operação de divisão ou multiplicação ou a combinação de ambas. É sobre o primeiro que tratarei na próxima seção.

\section{O campo conceitual aditivo ou, simplesmente, as estruturas aditivas}

A teoria dos campos conceituais (Vergnaud, 1990; 2009) afirma que a aquisição do conhecimento se dá por meio de situações e problemas já conhecidos, e que o conhecimento, portanto, tem características locais. Consequentemente, todos os conceitos têm um domínio de validade restrito, o qual varia de acordo com a experiência e com o desenvolvimento cognitivo do sujeito. A adição e a subtração são exemplos de conceitos, os quais não têm sentido se estudados isoladamente, mas sim dentro de um campo conceitual, o das estruturas aditivas.

Devido à grande diversidade de conceitos envolvidos nessas estruturas, elas fazem parte de um conhecimento que o aluno adquire a médio e a longo prazo, devendo ser proposto ao longo das quatro séries iniciais. As situações encontradas nas estruturas aditivas podem ser de tipos diversos (composição, transformação e comparação) e apresentam níveis de complexidade cognitiva, variando desde as consideradas prototípicas, até as de quarta extensão. A seguir apresento uma re-leitura da classificação proposta por Vergnaud (1982), feita por Magina, Nunes, Campos e Gitirana (2001).

Problemas de composição são aqueles em que duas partes se juntam para formar um todo, podendo a situação variar se o total e uma das partes for conhecidos e se perguntar sobre a outra parte. Em contextos de quantidades, por exemplo, situaçõesproblema do tipo "Há 4 meninos e 7 meninas em volta de uma mesa. Quantas crianças são ao todo?" são resolvidos com sucesso por crianças bem novas (entre 4 e 6 anos) e se enquadram no que Vergnaud (1982) chamou de problemas protótipos.

Problemas de transformação são aqueles que têm um estado inicial, uma transformação (positiva ou negativa) e um estado final. A situação-problema mais simples (também consideradas como prototípicas) é quando o estado inicial e a transformação ocorrida são conhecidos e pergunta-se pelo estado final. Nesse caso, crianças, a partir de 4 e 5 anos, já têm sucesso. Já as situações de transformação, com estado inicial desconhecido, são consideradas as mais difíceis. 
Problemas de comparação podem ser de relação estática entre dois todos - "Ana tem 8 anos. Carlos tem 12 anos. Quem tem mais anos? Quantos anos a mais?" - ou de relação dinâmica, quando é dada a relação entre dois todos e apenas um é conhecido - "Carlos tem 9 reais e Luiz tem 6 reais a mais que Carlos. Quantos reais tem Luiz?".

Podemos dizer que os problemas de composição são os mais simples. O Quadro 1 a seguir apresenta um resumo dessa classificação.

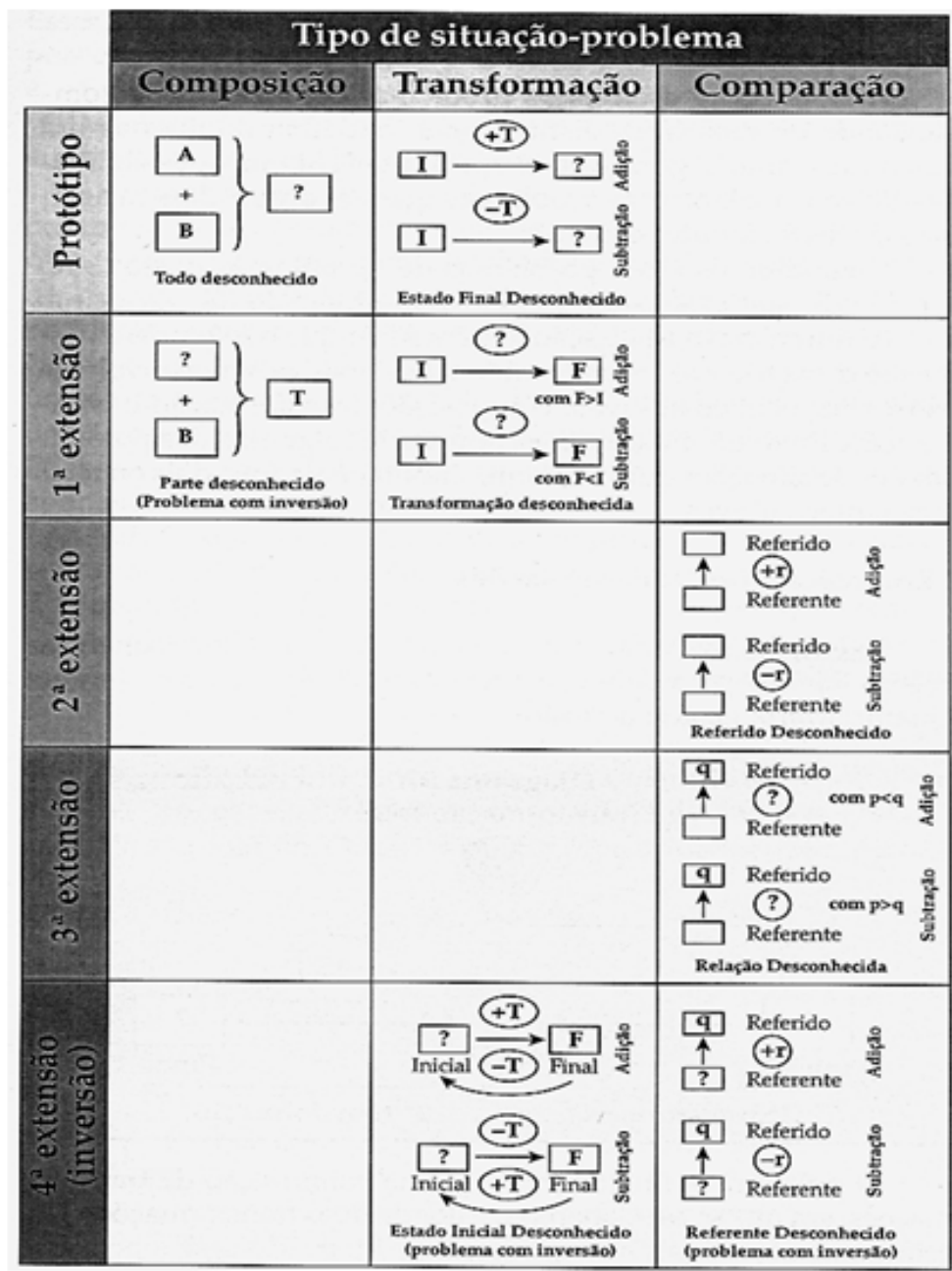

QUADRO 1 - CLASSIFICAÇAO DOS PROBLEMAS ADITIVOS

FONTE: Magina et al. (2001, p. 51). 
Segundo Magina et al. (2001), essa classificação oferece uma estrutura teórica que ajuda, tanto o pesquisador quanto o professor, a entender o significado das diferentes representações simbólicas da adição e subtração, além de servir de base para o cenário de experiências sobre esses processos matemáticos na sala de aula. Ela ainda contribui para que o professor possa compreender o amplo espectro de significações das operações, evidenciando a complexidade do trabalho a ser realizado para que os alunos ampliem a compreensão dos conceitos envolvidos nessas operações.

\section{O estudo}

Trata-se de um estudo de caráter descritivo, em que foi solicitado coletivamente a 103 professores que individualmente elaborassem e resolvessem oito problemas, quatro de estrutura aditiva e quatro de estrutura multiplicativa. Esses professores, todos em serviço, atuavam nas séries iniciais do ensino fundamental da rede pública estadual de São Paulo.

Neste artigo tratarei apenas dos resultados obtidos no instrumento diagnóstico relativo à elaboração de problemas de estruturas aditivas, ou seja, dos problemas elaborados no campo conceitual aditivo.

O objetivo aqui é o de saber até onde esses professores avançam em relação ao campo conceitual aditivo considerando a classificação de problemas anteriormente apresentados no Quadro 1. É sabido que, em geral, para além dos protótipos, os demais problemas da classificação precisam ser trabalhados pelos professores para que possam ser apreendidos pelos estudantes.

Construo a hipótese de que a maioria dos professores centra seu trabalho com os alunos das séries iniciais do Ensino Fundamental nos protótipos aditivos. O que muda quase sempre é o contexto e a magnitude do número. Ora, Magina et al. (2001) mostraram que os alunos, quando ingressam na escola, em geral, dominam os protótipos e terminam as séries iniciais sem dominar problemas em que o campo conceitual aditivo é estendido.

O primeiro resultado que saltou aos olhos foi que a quase totalidade dos problemas (344 do total de 389 problemas, ou seja, $88,43 \%$ do total de problemas elaborados) foi classificada como protótipos aditivos. Notava-se que os professores variavam os contextos dos problemas, bem como a magnitude dos números ou, ainda, a quantidade de parcelas a serem somadas. Os raciocínios exigidos nos problemas, contudo, eram de dois tipos: a) composição em que as partes são conhecidas e se perguntava pelo valor do todo; ou b) problemas 
imersos na situação de transformação, em que era dado um valor inicial, informado que certa quantidade era adicionada ou retirada desse tanto e perguntava-se pela quantidade final.

Os demais problemas elaborados (45) foram classificados entre a primeira $\mathrm{e}$ a terceira extensão (ver Quadro 1), sendo que nenhum deles chegou a representar um percentual de $4 \%$ dos problemas elaborados. Os problemas de composição, conhecendo-se uma das partes e o todo, foram os que mais apareceram depois dos protótipos ( $1 / 3$ desses problemas, o que representou um percentual 3,86\% da amostra total dos problemas), o que dá indício de que esses professores estão concentrando seu ensino das estruturas aditivas, sobremaneira, no trabalho com os protótipos.

\section{Conclusão}

Analisados os problemas elaborados pelos professores, a principal conclusão deste artigo é a seguinte: comparados os resultados desses problemas com os resultados obtidos pelos estudantes da 4. ${ }^{\text {a }}$ série do Ensino Fundamental, nos mais recentes instrumentos diagnósticos oficiais (BRASIL, 2006; 2008), podese ver que existe uma estreita relação entre o desempenho das crianças nesses diagnósticos e o tipo de problemas que os professores do estudo elaboraram. De fato, parece não haver uma preocupação, ou compreensão, dos professores em trabalhar problemas que facilitem o entendimento e a expansão do campo conceitual aditivo de seus estudantes.

Na sequência, sinto-me confortável para comprovar a minha hipótese de que a maioria dos professores centra seu trabalho com os alunos das séries iniciais do Ensino Fundamental nos problemas aditivos considerados como protótipos, mudando apenas o contexto em que se apresentam e/ou a magnitude dos números envolvidos.

Por fim, ao retomar a discussão do caráter interdisciplinar da educação matemática, é importante lembrar que o presente estudo, cujo conteúdo está inserido no campo da matemática, contou com o apoio da teoria psicológica de Vergnaud (1982; 1990; 1996; 1998; 2009), a qual auxiliou a investigar o que pode ser uma importante variável na formação do conceito aditivo dos estudantes nesse nível de escolarização.

A questão que se coloca, então, é a necessidade de se realizar pesquisas que busquem investigar questões como: essa aparente "falta de preocupação", de parte dos professores, passa pelo seu desconhecimento da teoria dos campos 
conceituais no que tange às estruturas aditivas? Ou, ainda, essa aparente "falta de preocupação" na verdade significa que os professores, tanto quanto os alunos avaliados pelo SAEB, também não têm seu campo conceitual aditivo expandido? Outras questões de caráter histórico - como traçar um panorama sobre o ensino das estruturas aditivas em décadas passadas - e também de caráter educacional o exame de currículos passados e leis de diretrizes curriculares, de documentos oficiais, da formação inicial dos professores polivalentes - podem ser realizadas por pedagogos e/ou psicólogos e/ou matemáticos. Assim, são citadas apenas algumas das áreas afins que podem (e obviamente serão bem-vindas) atuar no campo da educação matemática.

Toda essa gama de possíveis pesquisas, cujo olhar certamente variará segundo a formação do pesquisador que a esteja realizando, trará contribuições essenciais para que se possa efetivamente atuar na direção da melhoria do ensino de matemática, em nosso País e no mundo.

\section{REFERÊNCIAS}

BRASIL. Relatório SAEB - Matemática. Sistema de avaliação do ensino básico. Brasília: INEP, MEC, 2006.

. Relatório SAEB - Matemática. Sistema de avaliação do ensino básico. Brasília: INEP, MEC, 2008.

CARRAHER, T.; CARRAHER, D.; SCHLIEMANN, A. Na vida dez, na escola zero. 3. ed. São Paulo: Cortez, 1989.

D’AMBROSIO, U. Etnomatemática: um programa. Educação Matemática em Revista. SBEM, Blumenau, n. 1, p. 5-11, 1993.

. Etnomatemática: elo entre as tradições e a modernidade. Belo Horizonte: Autêntica, 2001.

FERNANDES, S. H. A.; HEALY, L. Educação matemática e inclusão: abrindo janelas teóricas para a aprendizagem de alunos cegos. Educação e Cultura Contemporânea, v. 5, p. 91-105, 2008.

GARNICA, V.; BARALDI, I. Traços e paisagens: a educação matemática nas décadas de 1960 e 1970. Bauru: Canal 6, 2005. v. 3. 
GARNICA, V.; SILVA, S. (Org.). Exercícios de pesquisa - Grupo de História Oral e Educação Matemática (GHOEM). Campo Grande: Universidade Federal do Mato Grosso do Sul, 2010. v. 1.

GIL, A. C. Como elaborar projetos de pesquisa. São Paulo: Atlas, 2002.

HEALY, L.; POZZI, S.; SUTHERLAND, R. Reflections on the role of the computer in the development of algebraic thinking. In: SUTHERLAND, R.; ROJANA, T.; BELL, A.; LINS, R. (Orgs.). Perspectives on School Algebra. Dordrecht: Kluwer Academic Publishers, 2001. p. 231-248.

HEALY, L.; SINCLAIR, N. If this is our mathematics, what are our stories? International Journal of Computers for Mathematical Learning, v. 12, n. 1, p. 3-21, 2007.

LAVE, J.; WENGER, E. Situated learning: Legitimate peripheral participation. New York: Cambridge University Press, 1998.

MACHADO, N. J. Matemática e Educação. 4. ed. São Paulo: Cortez Editora, 1992. v. 1. . Educación: Seis propuestas para el próximo milénio. Cuadernos de Investigación, v. 2, n. xxx, p. 25-38, 2003.

MAGINA, S.; CAMPOS, T. As estratégias dos alunos na resolução de problemas aditivos: um estudo diagnóstico. Educação Matemática Pesquisa, v. 6, n. 1, p. 53-71, 2004.

MAGINA, S.; CAMPOS, T. M. M.; NUNES, T.; GITIRANA, V. Repensando adição e subtração: contribuições da teoria dos campos conceituais. São Paulo: PROEM, 2001.

MEIRA, L. Atividade algébrica e produção de significados em matemática: um estudo de caso. In: DIAS, M. das G.; SPINILLO, A. G. (Orgs.). Tópicos em psicologia cognitiva. Recife: UFPE, 1996. p. 168-192.

. Mathematical representations as systems of notations-in-use. In: GRAVEMEIJER, K.; LEHRER, R.; van OERS, B.; VERSCHAFFEL, L. (Orgs.). Symbolizing, modeling and tool use in mathematics education. Dordrecht: Kluwer Academic Publishers, 2002. p. 87-103.

.Zonas de desenvolvimento proximal nas salas de aula de ciências e matemática. In: CASTRO, M. R. de. (Org.). VETOR: Série de Divulgação Científica em Educação Matemática. Campos: FAFIC, 2003, p. 5-23. v. 1.

NUNES, T.; BRYANT, P. Crianças Fazendo Matemática. Tradução de: COSTA, S. Porto Alegre: Artes Médicas, 1997. 
NUNES, T.; CAMPOS, T. M. M.; MAGINA, S.; BRYANT, P. Educação Matemática: números e operações numéricas. São Paulo: Cortez, 2005.

PIAGET, J. Abstração Reflexionante. Tradução de: BECKER F.; SILVA, B. G. da. Porto Alegre: Artes Médicas, 1995.

PIAGET, J. SZEMINSKA, A. A Gênese do Número na Criança. Tradução de: OITICICA, C. M. 2. ed. Rio de Janeiro: Zahar, 1975.

PIAGET, J.; INHELDER, B. A origem da idéia do acaso na criança. Tradução de: COELHO, A. M. Rio de Janeiro: Record, 1961.

. A Psicologia da Criança. Tradução de: CAJADO, O. M. 14. ed. Rio de Janeiro: Bertrand Brasil, 1995.

RUDIO, F. Introdução ao projeto de pesquisa científica. São Paulo: Vozes, 2009.

SPINILLO, A. O sentido de número e sua importância na educação matemática. In: BRITO, M. R. F. de (Org.). Solução de problemas e a matemática escolar. Campinas: Editora Alínea, 2006. p. 83-111.

SPINILLO, A.; MAGINA, S. Alguns 'mitos' sobre a educação matemática e suas conseqüências para o ensino fundamental. In: PAVANELLO, R. M. (Org.). Matemática nas Séries Iniciais do ensino Fundamental. São Paulo: Col. SBEM, 2004. p. 7-35.

VALENTE, W. (Org.). Euclides Roxo e a modernização do ensino da matemática no Brasil. 2. ed. Brasília: Editora da Universidade de Brasília, 2004.

VALENTE, W.; SILVA, M. C. Na oficina do historiador da educação matemática: cadernos de alunos como fontes de pesquisa. Belém: SBHMat, 2009.

VERGNAUD, G. A Classification of cognitive tasks and operations of thought involved in addition and subtraction problems. In: CARPENTER, T. P.; MOSER, J. M.; ROMBERG, T. A. (Eds.). Addition and subtraction: a cognitive perspective. Hillsdale NJ: Lawrence Erlbaun, 1982. p. 39-59.

. Epistemology and psychology of mathematics education. In: NESHER, P.; KILPATRICK, J. (Eds.). Mathematics and cognition A research synthesis by the International Group for the Psychology of Mathematics Education. Cambridge: Cambridge University Press, 1990. p. 14-30.

A teoria dos campos conceituais. In. BRUN, J. (Org.). Didáctica das matemáticas. Tradução de: FIGUEIREDO, M. J. Lisboa: Instituto Piaget, 1996. p. 155-191. 
. A Comprehensive Theory of Representation for Mathematics Education.

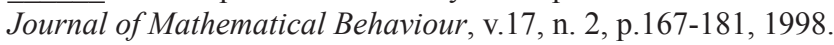

. A Criança, a Matemática e a Realidade. Tradução de: MORO, M. L. F. Curitiba: Editora UFPR, 2009. 
